\title{
Quantum Oscillator in a Blackbody Radiation Field II. Direct Calculation of the Energy Using the Fluctuation-Dissipation Theorem
}

\author{
G. W. FORD \\ Department of Physics, \\ The University of Michigan, Ann Arbor, Michigan 48109-1120 \\ J. T. LEWIS \\ School of Theoretical Physics, \\ Dublin Institute for Advanced Studies, Dublin 4, Ireland \\ AND \\ R. F. O’CONNELL \\ Department of Physics and Astronomy, \\ Louisiana State University, Baton Rouge, Louisiana 70803-4001 \\ Received October 19, 1987
}

\begin{abstract}
An earlier exact result (Phys. Rev. Lett. 55 (1985), 2273) for the free energy of an oscillatordipole interacting with the radiation field is obtained using the fluctuation-dissipation theorem. A key feature of the earlier calculation, a remarkable formula for the free energy of the oscillator, is obtained in the form of a corresponding formula for the oscillator energy. This confirms, by a longer, more conventional proof, the earlier result. An advantage of this present method is that separate contributions to the energy can be isolated and discussed. Explicit, closed-form expressions are given and the high-temperature limit is discussed. (1) 1988 Academic Press, Inc.
\end{abstract}

\section{INTRODUCTION}

In an earlier publication we presented an exact calculation of the free energy of an oscillator-dipole interacting with the radiation field in a blackbody cavity [1]. There we used novel methods, those of quantum stochastic processes, to derive a remarkable formula for the free energy of an oscillator with linear passive dissipation. We then applied the formula to get our result for the electromagnetic case. Although we gave a short rigorous proof of this formula, the notions and the method of proof were novel and possibly misunderstood by some readers. Our 
main purpose here, therefore, is to obtain our exact result by an alternative, more conventional, method using the fluctuation-dissipation theorem.

Our earlier result was somewhat surprising in that it predicts a high-temperature shift of the energy that is proportional to the square of the temperature and negative in sign. The magnitude of this shift is in agreement with that obtained by several earlier authors using a variety of methods, but, and this was the surprise, the sign is opposite. An advantage of this present method is that we can calculate explicitly separate contributions to the energy. A second purpose of this present paper, therefore, is to discuss in some detail these contributions and their relation to the exact result.

The remarkable formula for the free energy of the oscillator takes the form

$$
F_{\mathrm{O}}(T)=\frac{1}{\pi} \int_{0}^{x} d \omega f(\omega, T) \operatorname{Im}\left\{\frac{d \ln \left[\alpha\left(\omega+i 0^{+}\right)\right]}{d \omega}\right\}
$$

where $\alpha(\omega)$ is the oscillator susceptibility. In this formula $f(\omega, T)$ is the free energy (including zero-point energy) of a free oscillator of frequency $\omega$ :

$$
f(\omega, T)=k_{\mathrm{B}} T \ln \left[2 \sinh \left(h \omega / 2 k_{\mathrm{B}} T\right)\right] .
$$

The corresponding formula for the oscillator energy is

$$
\begin{aligned}
U_{\mathrm{O}}(T) & =F_{\mathrm{O}}(T)-T \partial F_{\mathrm{O}}(T) / \partial T \\
& =\frac{1}{\pi} \int_{0}^{\infty_{\alpha}} d \omega u(\omega, T) \operatorname{Im}\left\{\frac{d \ln \left[\alpha\left(\omega+i 0^{+}\right)\right]}{d \omega}\right\} .
\end{aligned}
$$

Here $u(\omega, T)$ is the Planck energy (including zero-point energy) of a free oscillator of frequency $\omega$ :

$$
u(\omega, T)=\frac{\hbar \omega}{2} \operatorname{coth}\left(\frac{\hbar \omega}{2 k_{\mathrm{B}} T}\right)
$$

In this paper we use the fluctuation-dissipation theorem to obtain the formula (1.3) in the special case of an oscillator interacting with the radiation field via dipole coupling.

In Section II we describe the appropriate Hamiltonian describing a one-dimensional oscillator in the radiation field and in Section III we use the fluctuation-dissipation theorem (described in the Appendix) to obtain an expression for the mean energy of the system. When we subtract the mean energy of the radiation field in the absence of the oscillator, we obtain our formula (1.3). The restriction to a one-dimensional oscillator is only a matter of convenience; results for a three-dimensional oscillator are obtained by multiplying by three. In Section IV, we evaluate these results to obtain explicit expressions for these quantities as well as the mean kinetic and potential energies and discuss in particular the hightemperature limit. 


\section{The Hamiltonian}

The Hamiltonian for a one-dimensional oscillator-dipole, with charge $-e$, interacting with the three-dimensional radiation field is $[2,3]$

$$
H_{0}=\frac{1}{2 m}\left(p+\frac{e}{c} A_{x}\right)^{2}+\frac{1}{2} K x^{2}+H_{\mathrm{R}},
$$

where $A_{x}$ is the component of the vector potential in the direction of the oscillator displacement, given by

$$
A_{x}=\sum_{\mathbf{k}, s}\left(2 \pi \hbar c^{2} / \omega_{k} V\right)^{1 / 2}\left(\hat{e}_{\mathbf{k}, s} \cdot \hat{x}\right)\left(f_{k}^{*} a_{\mathbf{k}, s}+f_{k} a_{\mathbf{k}, s}^{\dagger}\right),
$$

and $H_{\mathrm{R}}$ is the radiation field Hamiltonian, given by

$$
H_{\mathbf{R}}=\sum_{\mathbf{k}, s} \frac{1}{2} \hbar \omega_{k}\left(a_{\mathbf{k}, s}^{\dagger} a_{\mathbf{k}, s}+a_{\mathbf{k}, s} a_{\mathbf{k}, s}^{\dagger}\right)
$$

For convenience in (2.2) we have chosen the polarization vector $\hat{e}_{\mathbf{k}, s}$ to be real. In (2.2), $f_{k}$ is the electron form factor, which must be such that it is unity up to some large cutoff frequency $\Omega$, after which it falls rapidly to zero.

The fluctuation-dissipation theorem deals with Hermitian operator variables, and, therefore, it will be convenient to introduce canonical field variables $q_{\mathbf{k} . s}$ and $p_{\mathbf{k}, s}$ and write

$$
a_{\mathbf{k}, s}=\frac{f_{k}}{\left|f_{k}\right|} \frac{m_{k} \omega_{k} q_{\mathbf{k}, s}+i p_{\mathbf{k}, s}}{\left(2 m_{k} \hbar \omega_{k}\right)^{1 / 2}}
$$

with

$$
m_{k}=\frac{4 \pi e^{2}\left|f_{k}\right|^{2}}{\omega_{k}^{2} V}
$$

Then the radiation Hamiltonian (2.3) becomes

$$
H_{\mathrm{R}}=\sum_{\mathbf{k}, s}\left(\frac{1}{2 m_{k}} p_{\mathbf{k}, s}^{2}+\frac{1}{2} m_{k} \omega_{k}^{2} q_{\mathbf{k}, s}^{2}\right)
$$

and from (2.2) we get

$$
\frac{e}{c} A_{x}=\sum_{\mathbf{k}, s}\left(\hat{e}_{\mathbf{k}, s} \cdot \hat{x}\right) m_{k} \omega_{k} q_{\mathbf{k}, s}
$$




\section{The MeAn Energy}

In the Appendix we state the fluctuation-dissipation theorem. In applying this theorem we are led to consider the Hamiltonian

$$
H=H_{0}-x f(t)-\sum_{\mathbf{k} . s} q_{\mathbf{k}, s} f_{\mathbf{k}, s}(t),
$$

where $f(t)$ and $f_{\mathbf{k}, s}(t)$ are $c$-number functions of time. The Heisenberg equations of motion with respect to this Hamiltonian are

$$
\begin{gathered}
\dot{x}=\frac{1}{m}\left(p+\frac{e}{c} A_{x}\right), \quad \dot{p}=-K x+f(t), \\
\dot{q}_{\mathbf{k} . s}=\frac{1}{m_{\mathbf{k}, s}} p_{\mathbf{k}, s}, \quad \dot{p}_{\mathbf{k}, s}=-m_{k} \omega_{k}^{2} q_{\mathbf{k}, s}+\left(\hat{e}_{\mathbf{k}, s} \cdot \hat{x}\right) m_{k} \omega_{k} \dot{x}+f_{\mathbf{k}, s}(t) .
\end{gathered}
$$

Fliminating the momentum variables, we can write these as

$$
\begin{array}{r}
m \ddot{x}+K x-\frac{e}{c} \dot{A}_{x}=f(t), \\
m_{k} \ddot{q}_{\mathbf{k}, s}+m_{k} \omega_{k}^{2} q_{\mathbf{k}, s}+\left(\hat{e}_{\mathbf{k}, s} \cdot \hat{x}\right) m_{k} \omega_{k} \dot{x}=f_{\mathbf{k}, s}(t) .
\end{array}
$$

Assume now a time dependence $f(t)=f(\omega) \exp (-i \omega t)$ and correspondingly for the other variables. Then these equations become

$$
\begin{gathered}
\left(-m \omega^{2}+K\right) \tilde{x}+i \omega \frac{e}{c} \tilde{A}_{x}=\tilde{f} \\
m_{k}\left(-\omega^{2}+\omega_{k}^{2}\right) \tilde{q}_{\mathbf{k}, s}-i \omega\left(\hat{e}_{\mathbf{k}, s} \cdot \hat{x}\right) m_{k} \omega_{k} \tilde{x}=\widetilde{f}_{\mathbf{k}, s} .
\end{gathered}
$$

This is a set of coupled linear equations for $\tilde{x}$ and the $\tilde{q}_{\mathbf{k} . s}$. The solution can be written in the form

$$
\begin{gathered}
\tilde{x}=\alpha(\omega) \tilde{f}+\sum_{\mathbf{k}, s} \alpha_{\mathbf{k}, s} \widetilde{f}_{\mathbf{k}, s}, \\
\tilde{q}_{\mathbf{k}, s}=-\alpha_{\mathbf{k}, s} \tilde{f}+\sum_{\mathbf{k}^{\prime}, s^{\prime}} \alpha_{\mathbf{k}, s, \mathbf{k}^{\prime}, s^{\prime}} \widetilde{f}_{\mathbf{k}, s},
\end{gathered}
$$

where

$$
\alpha(\omega)=\left[-m \omega^{2}+K+\sum_{\mathbf{k}, s}\left(\hat{e}_{\mathbf{k}, s} \cdot \hat{x}\right)^{2} m_{k} \omega_{k}^{2} \frac{\omega^{2}}{\omega^{2}-\omega_{k}^{2}}\right]^{-1}
$$

is the oscillator susceptibility,

$$
\alpha_{\mathbf{k}, s}=\left(\hat{e}_{\mathbf{k}, s} \cdot \hat{x}\right) \frac{i \omega \omega_{k}}{\omega^{2}-\omega_{k}^{2}} \alpha(\omega)
$$


could be called the cross-susceptibility, and

$$
\begin{aligned}
\alpha_{\mathbf{k}, s ; \mathbf{k}^{\prime}, s^{\prime}}= & \left(\hat{e}_{\mathbf{k}, s} \cdot \hat{x}\right)\left(\hat{e}_{k^{\prime}, s^{\prime}} \cdot \hat{x}\right) \frac{\omega^{2} \omega_{k} \omega_{k^{\prime}}}{\left(\omega^{2}-\omega_{k}^{2}\right)\left(\omega^{2}-\omega_{k^{\prime}}^{2}\right)} \alpha(\omega) \\
& -\frac{1}{m_{k}\left(\omega^{2}-\omega_{k}^{2}\right)} \delta_{\mathbf{k}, \mathbf{k}^{\prime}} \delta_{s, s^{\prime}}
\end{aligned}
$$

is the field-oscillator susceptibility. These are the response functions we need in order to apply the fluctuation-dissipation theorem.

The fluctuation-dissipation theorem (A.16) tells us immediately that

$$
\begin{aligned}
\Psi\left(t-t^{\prime}\right) & \equiv \frac{1}{2}\left\langle x(t) x\left(t^{\prime}\right)+x\left(t^{\prime}\right) x(t)\right\rangle \\
& =\frac{h}{\pi} \int_{0}^{\infty} d \omega \operatorname{coth}\left(\frac{h \omega}{2 k_{\mathrm{B}} T}\right) \operatorname{Im}\left\{\alpha\left(\omega+i 0^{+}\right)\right\} \cos \omega\left(t-t^{\prime}\right)
\end{aligned}
$$

and that

$$
\begin{aligned}
\Psi_{\mathbf{k}, s ; \mathbf{k}, s} & \equiv \frac{1}{2}\left\langle q_{\mathbf{k}, s}(t) q_{\mathbf{k}, s}\left(t^{\prime}\right)+q_{\mathbf{k}, s}\left(t^{\prime}\right) q_{\mathbf{k}, s}(t)\right\rangle \\
& =\frac{\hbar}{\pi} \int_{0}^{\infty} d \omega \operatorname{coth}\left(\frac{\hbar \omega}{2 k_{\mathbf{B}} T}\right) \operatorname{Im}\left\{\alpha_{\mathbf{k}, s ; \mathbf{k}, s}\left(\omega+i 0^{+}\right)\right\} \cos \omega\left(t-t^{\prime}\right) .
\end{aligned}
$$

The mean of the Hamiltonian (2.1) is

$$
\begin{aligned}
U(T) & =\left\langle H_{0}\right\rangle \\
& =\left\langle\frac{1}{2} m \dot{x}^{2}+\frac{1}{2} K x^{2}+\sum_{\mathbf{k}, s}\left(\frac{1}{2} m_{k} \dot{q}_{\mathbf{k}, s}^{2}+\frac{1}{2} m_{k} \omega_{k}^{2} q_{\mathbf{k}, s}^{2}\right)\right\rangle .
\end{aligned}
$$

Here we have used the expression (2.6) for $H_{\mathrm{R}}$ and then eliminated the momentum variables using the equations of motion (3.2). Consider first the mean of the free oscillator Hamiltonian:

$$
\left\langle\frac{1}{2} m \dot{x}^{2}+\frac{1}{2} K x^{2}\right\rangle=\frac{\hbar}{\pi} \int_{0}^{\infty} d \omega \operatorname{coth}\left(\frac{\hbar \omega}{2 k_{\mathrm{B}} T}\right)\left(\frac{m \omega^{2}}{2}+\frac{K}{2}\right) \operatorname{Im}\left\{\alpha\left(\omega+i 0^{+}\right)\right\} .
$$

This result is obtained using the expression for $\left\langle x^{2}\right\rangle$ obtained from (3.9) by setting $t^{\prime}$ equal to $t$, and the expression for $\left\langle\dot{x}^{2}\right\rangle$ obtained from the same equation by first differentiating with respect to $t$ and $t^{\prime}$ and then setting $t^{\prime}$ equal to $t$. 
Next consider the expression for the mean of the radiation field Hamiltonian:

$$
\begin{aligned}
\left\langle H_{\mathrm{R}}\right\rangle & =\left\langle\sum_{\mathbf{k}, s}\left(\frac{1}{2} m_{k} \dot{q}_{\mathbf{k}, s}^{2}+\frac{1}{2} m_{k}\left(\omega_{k}^{2} q_{\mathbf{k}, s}^{2}\right)\right\rangle\right. \\
& =\frac{h}{\pi} \int_{0}^{\infty} d \omega \operatorname{coth}\left(\frac{\hbar \omega}{2 k_{\mathrm{B}} T}\right) \sum_{\mathbf{k}, s} \frac{1}{2} m_{k}\left(\omega^{2}+\omega_{k}^{2}\right) \operatorname{Im}\left\{\alpha_{\mathbf{k}, s ; \mathbf{k}, s}\left(\omega+i 0^{+}\right)\right\} .
\end{aligned}
$$

This result is obtained from (3.10) in the same way that the result (3.12) was obtained from (3.9). Using now the expression (3.8) for the field-oscillator susceptibility, we can write this result in the form

$$
\begin{aligned}
\left\langle H_{\mathrm{R}}\right\rangle= & \frac{h}{2 \pi} \int_{0}^{x} d \omega \operatorname{coth}\left(\frac{\hbar \omega}{2 k_{\mathrm{B}} T}\right) \operatorname{Im}\left\{-\sum_{\mathbf{k} . s} \frac{\omega^{2}+\omega_{k}^{2}}{\left(\omega+i 0^{+}\right)^{2}-\omega_{k}^{2}}\right. \\
& \left.+\omega^{2} \sum_{\mathbf{k}, s}\left(\hat{e}_{\mathbf{k} . s} \cdot \hat{x}\right)^{2} m_{k} \omega_{k}^{2} \frac{\omega^{2}+\omega_{k}^{2}}{\left[\left(\omega+i 0^{+}\right)^{2} \cdot \omega_{k}^{2}\right]^{2}} \alpha\left(\omega+i 0^{+}\right)\right\} .
\end{aligned}
$$

Here in the first sum within the braces we note that, since $\omega$ is positive within the range of integration,

$$
\frac{\omega^{2}+\omega_{k}^{2}}{\left(\omega+i 0^{+}\right)^{2}-\omega_{k}^{2}}=\frac{\omega^{2}+\omega_{k}^{2}}{\left(\omega+\omega_{k}\right)\left(\omega-\omega_{k}+i 0^{+}\right)}=\frac{\omega^{2}+\omega_{k}^{2}}{\omega+\omega_{k}}\left[P \frac{1}{\omega-\omega_{k}}-i \pi \delta\left(\omega-\omega_{k}\right)\right] .
$$

Hence, when we form the imaginary part, this sum contributes

$$
-\frac{h}{2 \pi} \int_{0}^{x_{0}} d \omega \operatorname{coth}\left(\frac{h \omega}{2 k_{\mathrm{B}} T}\right) \operatorname{Im}\left\{\sum_{\mathbf{k}, s} \frac{\omega^{2}+\omega_{k}^{2}}{\left(\omega+i 0^{+}\right)^{2}-\omega_{k}^{2}}\right\}=\sum_{\mathbf{k}, s} u\left(\omega_{k}, T\right),
$$

where $u(\omega, T)$, given by (1.4), is the Planck energy. This sum is just the mean energy of the free radiation field in the absence of the oscillator:

$$
\begin{aligned}
U_{\mathrm{R}} & \equiv \operatorname{Tr}\left\{H_{\mathrm{R}} \exp \left(-H_{\mathrm{R}} / k_{\mathrm{B}} T\right)\right\} / \operatorname{Tr}\left\{\exp \left(-H_{\mathrm{R}} / k_{\mathrm{B}} T\right)\right\} \\
& =\sum_{\mathbf{k}, s} u\left(\omega_{k}, T\right) .
\end{aligned}
$$

Using these results, (3.13) becomes

$$
\begin{aligned}
\left\langle H_{\mathrm{R}}\right\rangle= & U_{\mathrm{R}}+\frac{\hbar}{2 \pi} \int_{0}^{\infty} d \omega \operatorname{coth}\left(\frac{\hbar \omega}{2 k_{\mathrm{B}} T}\right) \\
& \times \operatorname{Im}\left\{\omega^{2} \sum_{\mathbf{k}, s}\left(\hat{e}_{\mathbf{k}, s} \cdot \hat{x}\right)^{2} m_{k} \omega_{k}^{2} \frac{\omega^{2}+\omega_{k}^{2}}{\left[\left(\omega+i 0^{+}\right)^{2}-\omega_{k}^{2}\right]^{2}} \alpha\left(\omega+i 0^{+}\right)\right\} .
\end{aligned}
$$


Now we can write the expression (3.6) for $\alpha(\omega)$ in the form

$$
\alpha(\omega)=\left[-m \omega^{2}+K-i \omega \tilde{\mu}(\omega)\right]^{-1},
$$

where

$$
\tilde{\mu}(\omega)=\sum_{\mathbf{k}, s}\left(\hat{\boldsymbol{e}}_{\mathbf{k} . s} \cdot \hat{x}\right)^{2} m_{k} \omega_{k}^{2} \frac{i \omega}{\omega^{2}-\overline{\omega_{k}^{2}}} .
$$

If we form

$$
\frac{d \tilde{\mu}(\omega)}{d \omega}=-i \sum_{\mathbf{k},:}\left(\hat{e}_{\mathbf{k}, s} \cdot \hat{x}\right)^{2} m_{k} \omega_{k}^{2} \frac{\omega^{2}+\omega_{k}^{2}}{\left(\omega^{2}-\omega_{k}^{2}\right)^{2}}
$$

and compare with (3.18), we see that we can write

$$
\left\langle H_{\mathrm{R}}\right\rangle=U_{\mathrm{R}}+\frac{\hbar}{2 \pi} \int_{0}^{\alpha} d \omega \operatorname{coth}\left(\frac{\hbar \omega}{2 k_{\mathrm{B}} T}\right) \operatorname{Im}\left\{i \omega^{2} \frac{d \tilde{\mu}\left(\omega+i 0^{+}\right)}{d \omega} \alpha\left(\omega+i 0^{+}\right)\right\} .
$$

The oscillator energy is defined to be the mean energy of the system of the oscillator interacting with the radiation field minus the mean energy of the radiation field in the absence of the oscillator:

$$
U_{\mathrm{O}}(T)=U(T)-U_{\mathrm{R}}(T) .
$$

Combining the results (3.11), (3.12), and (3.22), we find

$$
U_{\mathrm{O}}(T)=\frac{\hbar}{2 \pi} \int_{0}^{\infty} d \omega \operatorname{coth}\left(\frac{\hbar \omega}{2 k_{\mathrm{B}} T}\right) \operatorname{Im}\left\{\left[m \omega^{2}+K+i \omega^{2} \frac{d \tilde{\mu}\left(\omega+i 0^{+}\right)}{d \omega}\right] \alpha\left(\omega+i 0^{+}\right)\right\} .
$$

Using the form (3.19) for $\alpha(\omega)$, it is a simple matter to see that

$$
\begin{aligned}
{\left[m \omega^{2}+K+i \omega^{2} \frac{d \tilde{\mu}(\omega)}{d \omega}\right] \alpha(\omega) } & =1+\frac{m \omega^{2}+i \omega \tilde{\mu}(\omega)+i \omega^{2}(d \tilde{\mu}(\omega) / d \omega)}{-m \omega^{2}+K-i \omega \tilde{\mu}(\omega)} \\
& =1+\omega \frac{d \ln \alpha(\omega)}{d \omega} .
\end{aligned}
$$

Putting this into (3.24) we obtain the remarkable formula (1.3) for the energy.

\section{Evaluation of the Mean Energy}

At $T=0$ the formula (1.3) for the oscillator encrgy becomes

$$
U_{\mathrm{O}}(0)=\frac{h}{2 \pi} \int_{0}^{\infty} d \omega \omega \operatorname{Im}\left\{\frac{d \ln \left[\alpha\left(\omega+i 0^{+}\right)\right]}{d \omega}\right\} .
$$


The free energy, of course, has the same value. This is a divergent (i.e., cutoff dependent) quantity without physical significance, since it can be removed by a choice of the zero of energy. We therefore consider only the temperature-dependent part of the energy:

$$
U_{\mathrm{O}}(T)-U_{\mathrm{O}}(0)=\frac{1}{\pi} \int_{0}^{x} d \omega \frac{h \omega}{\exp \left(\hbar \omega / k_{\mathrm{B}} T\right)-1} \operatorname{Im}\left\{\frac{d \ln \left[\alpha\left(\omega+i 0^{+}\right)\right]}{d \omega}\right\} .
$$

In previous publications $[1,4]$ we have shown that in the limit of infinite volume of the blackbody radiation cavity, $V \rightarrow \infty$, and in the limit of large cutoff $(3.20)$ becomes

$$
\tilde{\mu}(\omega)=-i \omega M /\left(1-i \omega \tau_{e}\right),
$$

where $M$ is the renormalized mass and

$$
\tau_{c}=2 e^{2} / 3 M c^{3} .
$$

In this same limit the restmass vanishes, $m \rightarrow 0$, and the susceptibility (3.19) becomes

$$
\alpha(\omega)=\left[-M \omega^{2} /\left(1-i \omega \tau_{e}\right)+K\right]^{-1} .
$$

Note that the force-constant of the oscillator is unchanged. There is no renormalization of the potential energy. The "renormalization of the frequency" introduced in some discussions, which is in fact a renormalization of $K$, is not the renormalization procedure of quantum electrodynamics. The point is that there is only a renormalization of particle parameters, e.g., mass or charge, not the external potential.

The susceptibility (4.5) can also be written in the form

$$
\alpha(\omega)=\frac{1-i \omega \tau_{e}}{M\left(-\omega^{2}+\omega_{0}^{2}-i \omega \gamma\right)},
$$

where

$$
\omega_{0}^{2}=K / M, \quad \gamma=\omega_{0}^{2} \tau_{\varepsilon}
$$

With this form, we see readily that (4.2) becomes

$$
U_{\mathrm{O}}(T)-U_{\mathrm{O}}(0)=\frac{1}{\pi} \int_{0}^{\infty} d \omega \frac{\hbar \omega}{\exp \left(\hbar \omega / k_{\mathrm{B}} T\right)-1}\left\{-\frac{\tau_{e}}{1+\omega^{2} \tau_{e}^{2}}+\frac{\left(\omega^{2}+\omega_{0}^{2}\right) \gamma}{\left(\omega^{2}-\omega_{0}^{2}\right)^{2}+\omega^{2} \gamma^{2}}\right\} .
$$

Now in general $\tau_{e}$ is a very short time; if $e$ and $M$ are the electron charge and mass, then $\tau_{e} \simeq 2 \times 10^{-24} \mathrm{sec}$. Therefore, we may in general require that $\hbar / k_{\mathrm{B}} T>>\tau_{e}$. 
Then in the first term within the braces in (4.8) we may neglect $\omega^{2} \tau_{e}^{2}$ in the denominator to get

$$
U_{\mathrm{O}}(T)-U_{\mathrm{O}}(0)=-\frac{\pi \tau_{e}\left(k_{\mathrm{B}} T\right)^{2}}{6 \hbar}+\frac{1}{\pi} \int_{0}^{\infty} d \omega \frac{\hbar \omega}{\exp \left(h \omega / k_{\mathrm{B}} T\right)-1} \frac{\left(\omega^{2}+\omega_{0}^{2}\right) \gamma}{\left(\omega^{2}-\omega_{0}^{2}\right)^{2}+\omega^{2} \gamma^{2}} .
$$

The remaining integral is exactly what one would get if in (4.2) one used in place of (4.6),

$$
\alpha_{D}(\omega)=\frac{1}{M\left(-\omega^{2}+\omega_{0}^{2}-i \omega \gamma\right)},
$$

which is the susceptibility corresponding to the familiar phenomenological DrudeLorentz model of dissipation (Ohmic friction) with friction constant $\zeta=M \gamma$. It may be of some interest that this integral can also be expressed in closed form. Thus, if we make the change of variables

$$
y=\frac{\hbar \omega}{2 \pi k_{\mathrm{B}} T}, \quad z=\frac{\hbar}{2 \pi k_{\mathrm{B}} T}\left[\frac{\gamma}{2}+i\left(\omega_{0}^{2}-\frac{\gamma^{2}}{4}\right)^{1 / 2}\right],
$$

then (4.9) can be written [5]

$$
\begin{aligned}
U_{\mathrm{O}}(T)-U_{\mathrm{O}}(0) & =-\frac{\pi \tau_{e}\left(k_{\mathrm{B}} T\right)^{2}}{6 h}+4 k_{\mathrm{B}} T \operatorname{Re}\left\{z \int_{0}^{\infty} d y y\left(y^{2}+z^{2}\right)^{-1}\left(e^{2 \pi y}-1\right)^{-1}\right\} \\
& =-\frac{\pi \tau_{e}\left(k_{\mathrm{B}} T\right)^{2}}{6 h}+k_{\mathrm{B}} T \operatorname{Re}\{2 z \ln z-2 z \psi(z)-1\},
\end{aligned}
$$

where $\psi(z)=d \ln \Gamma(z) / d z$ is the logarithmic derivative of the gamma function. In the high-temperature limit, $k_{\mathrm{B}} T \gg \hbar \omega_{0}$, this expression becomes

$$
U_{\mathrm{O}}(T)-U_{\mathrm{O}}(0) \sim-\frac{\pi \tau_{e}\left(k_{\mathrm{B}} T\right)^{2}}{6 h}+k_{\mathrm{B}} T-\frac{\hbar \gamma}{2 \pi} \ln \frac{\pi k_{\mathrm{B}} T}{h \omega_{0}}+\cdots
$$

The leading term is negative. This is the surprising result we first obtained in Ref. [1], a result opposite in sign to that obtained by a considerable number of previous investigators. The second term, $k_{\mathrm{B}} T$, is the familiar equipartition energy and, because of the smallness of $\tau_{e}$, is much the larger term.

Some understanding of the apparently paradoxical sign of the high-temperature $T^{2}$ term is obtained if we form the vibrational kinetic energy

$$
\begin{aligned}
K E(T) & =\left\langle\frac{1}{2} M \dot{x}^{2}\right\rangle \\
& =\frac{\hbar}{2 \pi} \int_{0}^{\infty} d \omega \operatorname{coth}\left(\frac{\hbar \omega}{2 k_{\mathrm{B}} T}\right) M \omega^{2} \operatorname{Im}\left\{\alpha\left(\omega+i 0^{+}\right)\right\} .
\end{aligned}
$$


This is obtained from (3.9) just as was (3.12). We stress that this vibrational kinetic energy is formed with the renormalized (observed) mass. In the limit we are considering the bare mass is zero and the renormalized mass is entirely of electromagnetic origin. Forming now the temperature-dependent part of this expression and using the form (4.6) for $\alpha(\omega)$, we find

$$
\begin{aligned}
K E(T)-K E(0) & =\frac{\hbar}{\pi} \int_{0}^{\infty} d \omega \frac{1}{\exp \left(\hbar \omega / k_{\mathrm{B}} T\right)-1} \frac{\omega^{5} \tau_{c}}{\left(\omega^{2}-\omega_{0}^{2}\right)^{2}+\omega^{2} \gamma^{2}} \\
& =\frac{\pi \tau_{e}\left(k_{\mathrm{B}} T\right)^{2}}{6 \hbar}-k_{\mathrm{B}} T \operatorname{Re}\left\{\frac{z^{2}}{z^{*}\left(z-z^{*}\right)}[2 z \ln z-2 z \psi(z)-1]\right\},
\end{aligned}
$$

where $z$ is again given by (4.11). In the high-temperature limit this becomes

$$
K E(T)-K E(0) \sim \frac{\pi \tau_{e}\left(k_{\mathrm{B}} T\right)^{2}}{6 \hbar}+\frac{1}{2} k_{\mathrm{B}} T\left(1-\gamma \tau_{e}\right)-\frac{\hbar \gamma}{\pi}\left(1-\frac{\gamma \tau_{e}}{2}\right) \ln \frac{2 \pi k_{\mathrm{B}} T}{h \omega}+\cdots
$$

The leading term here is the same as in (4.13), but now it is positive, as it must be. The second term is the equipartition kinetic energy, with a negligibly small correction due to the finite width of the quantum levels.

The mean of the oscillator potential energy is

$$
\begin{aligned}
P E(T) & =\left\langle\frac{1}{2} K x^{2}\right\rangle \\
& =\frac{\hbar K}{2 \pi} \int_{0}^{x} d \omega \operatorname{coth}\left(\frac{\hbar \omega}{2 k_{\mathrm{B}} T}\right) \operatorname{Im}\left\{\alpha\left(\omega+i 0^{+}\right)\right\} .
\end{aligned}
$$

Note that this is equal to the mean (3.12) of the unrenormalized free oscillator Hamiltonian, since the difference is $K E(T)$ multiplied by the ratio $m / M$, which is zero. Forming the temperature-dependent part and using the form (4.6) for $\alpha(\omega)$, we find

$$
\begin{aligned}
P E(T)-P E(0) & =\frac{\hbar}{\pi} \int_{0}^{\infty} d \omega \frac{1}{\exp \left(\hbar \omega / k_{\mathrm{B}} T\right)-1} \frac{\omega^{3} \gamma}{\left(\omega^{2}-\omega_{0}^{2}\right)^{2}+\omega^{2} \gamma^{2}} \\
& =k_{\mathrm{B}} T \operatorname{Re}\left\{\frac{z}{z-z^{*}}[2 z \ln z-2 z \psi(z)-1]\right\} \\
& \sim \frac{1}{2} k_{\mathrm{B}} T-\frac{\hbar \gamma}{2 \pi} \ln \frac{2 \pi k_{\mathrm{B}} T}{\hbar \omega}+\cdots .
\end{aligned}
$$

Here the leading term is the equipartition potential energy.

As we have noted, the mean potential energy can be isolated in the expression (3.11) for the mean of the Hamiltonian of the system of oscillator plus bath,

$$
U(T)=P E(T)+\left\langle H_{\mathrm{R}}\right\rangle,
$$


but the mean kinetic energy is part of $\left\langle H_{\mathrm{R}}\right\rangle$; it is of electromagnetic origin. One might be tempted to take $E(T)=K E(T)+P E(T)$ to be the oscillator energy, but this ignores what one can call the energy of interaction with the electromagnetic field. In the high-temperature limit this energy of interaction is negative, equal to twice the kinetic energy.

The entropy associated with the oscillator can be obtained from the familiar thermodynamic relation between free energy, energy, and entropy: $F=U-T S$. Since the high-temperature $T^{2}$ dependence of the oscillator energy and free energy have opposite signs, we see immediately that

$$
S_{\mathrm{O}}(T) \sim-\frac{\pi \tau_{e} k_{\mathrm{B}}^{2} T}{h}+\cdots .
$$

How does one understand this negative entropy associated with the presence of the oscillator? We think the way to say it is that the oscillator "loads" the normal modes of the cavity, thereby reducing their fluctuations and the corresponding entropy. Support for this view is given by the perturbative calculation of Barton [7].

The positive sign of the $T^{2}$ contribution to the mean kinetic energy explains the crror made by at least some of the earlier investigators: they thought they were caculating $U_{\mathrm{O}}(T)$, but in fact the quantity calculated was $K E(T)$. As we have argued, it is the free energy $F_{\mathrm{O}}(T)$ which corresponds to the measured quantity $[1,6]$. As we see from $(1.3)$, a negative $T^{2}$ term in $U_{O}(T)$ implies a positive $T^{2}$ term in $F_{\mathrm{O}}(T)$.

\section{APPENDiX: The Fluctuation-Dissipation Theorem}

The roots of the fluctuation-dissipation theorem can be traced back through Onsager and Nyquist to Einstein, and perhaps beyond. However, the precisely stated mechanical form we use first appeared in the classic paper of Callen and Welton [8]. By now the theorem is familiar to every educated physicist, but its very familiarity has spawned so many versions and such a variety of terminology that we feel it necessary to state the theorem once again. That is the purpose of this appendix. For a modern textbook in which the theorem is discussed and proved, see Landau and Lifshitz [9]. A more formal pruof along the lines of our statement is given by Case [10].

The theorem is very general. It has to do with a system with Hamiltonian $H_{0}$, which is otherwise unspecified. (A Hamiltonian must be a Hermitian operator with a spectrum bounded from below. The lower bound must be an eigenvalue, and the corresponding eigenvector is the ground state.) If the system is in equilibrium at temperature $T$, then the thermal expectation of an operator $\theta$ is defined to be

$$
\langle c\rangle=\operatorname{Tr}\left\{\left(\mathcal{e x p}\left(-H_{0} / k_{\mathrm{B}} T\right)\right\} / \operatorname{Tr}\left\{\exp \left(-H_{0} / k_{\mathrm{B}} T\right)\right\} .\right.
$$


The Heisenberg operator at time $t$ which is equal to the operator 0 at time $t=0$ is

$$
t(t)=\exp \left(i H_{0} t / \hbar\right)\left(c \exp \left(-i H_{0} t / \hbar\right)\right.
$$

These are the basic notions required for the statement of the theorem.

Consider a set of operators for the system $y_{1}, y_{2}, \ldots$. For simplicity we assume

$$
\left\langle y_{i}\right\rangle=0 ; \quad j=1,2, \ldots
$$

The correlation functions are defined to be

$$
\psi_{j . k}\left(t-t^{\prime}\right) \equiv \frac{1}{2}\left\langle y_{j}(t) y_{k}\left(t^{\prime}\right)+y_{k}\left(t^{\prime}\right) y_{j}(t)\right\rangle \text {. }
$$

Next consider the perturbed time-dependent Hamiltonian

$$
H(t)=H_{0}-\sum_{i} y_{i} f(t)
$$

where $f_{j}(t) ; j=1,2, \ldots$; are real $c$-number functions of time (generalized applied forces) which vanish in the distant past,

$$
\operatorname{Lim}_{t \rightarrow-\infty} f_{j}(t)=0
$$

It is assumed that in the distant past $(t=-\infty)$ the system is in equilibrium under the Hamiltonian $H_{0}$ and one considers the subsequent development under the perturbed Hamiltonian $H$. This time development corresponds to the unitary transformation $U(t)$ that is the solution of the equation

$$
i n \frac{\partial U}{\partial t}=H(t) U
$$

subject to the condition in the distant past,

$$
\operatorname{Lim}_{t \rightarrow-\infty} U(t) \exp \left(i H_{0} t / \hbar\right)=1
$$

One then introduces

$$
y_{j}^{(r)}(t) \equiv\left\langle U^{+}(t) y_{j} U(t)\right\rangle
$$

which is the thermal expectation of the operator $y_{j}$ developing in time under the action of the applied forces $f_{1}(t), f_{2}(t), \ldots$. If these forces are weak one may expand,

$$
y_{j}^{(r)}(t)=\sum_{k} \int_{-\varsigma}^{t} d t^{\prime} \alpha_{j, k}\left(t-t^{\prime}\right) f_{k}\left(t^{\prime}\right),
$$

neglecting terms higher than linear in the forces. The functions $\alpha_{j, k}(t)$ are the linear 
response functions, also called the generalized susceptibilities. In terms of the operators $y_{j}$, one can use perturbation theory to show

$$
\alpha_{j, k}(t)=\frac{i}{\hbar}\left\langle\left[y_{j}(t), y_{j}\right]\right\rangle \theta(t)
$$

where $\theta(t)$ is the Heaviside function.

One now introduces the Fourier transform of the correlation functions,

$$
\tilde{\psi}_{j, k}(\omega)=\int_{-\infty}^{\infty} d t e^{i \omega t} \psi_{j, k}(t)
$$

The Fourier transform of the response function is a bit special:

$$
\bar{\alpha}_{j, k}(\omega)=\int_{0}^{\infty} d t e^{i z t} x_{j, k}(t), \quad \operatorname{Im} z>0 .
$$

This is clearly analytic in the upper half $z$-plane. On the real axis one must cvaluatc the limit as $z$ approaches real $\omega$ from above, $z \rightarrow \omega+i 0^{+}$. This corresponds to the condition (A.8).

With these preliminaries we can now state the fluctuation-dissipation theorem:

$$
\Psi_{j, k}(\omega)=\frac{\hbar}{2 i} \operatorname{coth}\left(\frac{\hbar \omega}{2 k_{\mathrm{B}} T}\right)\left[\tilde{\alpha}_{j, k}\left(\omega+i 0^{+}\right)-\tilde{\alpha}_{k, j}\left(\omega+i 0^{+}\right)^{*}\right]
$$

If $k=j$ the quantity in brackets is

$$
\tilde{\alpha}_{j, j}\left(\omega+i 0^{+}\right)-\tilde{\alpha}_{j, j}\left(\omega+i 0^{+}\right)^{*}=2 i \operatorname{Im}\left\{\tilde{\alpha}_{j, j}\left(\omega+i 0^{+}\right)\right\} .
$$

The reality condition requires that this be an odd function of $\omega$. Therefore, for this case, forming the inverse Fourier transform, the fluctuation-dissipation theorem can be written

$$
\psi_{j, j}(t)=\frac{\hbar}{\pi} \int_{0}^{\infty} d \omega \operatorname{coth}\left(\frac{\hbar \omega}{2 k_{\mathrm{B}} T}\right) \operatorname{Im}\left\{\tilde{\alpha}_{j, j}\left(\omega+i 0^{+}\right)\right\} \cos \omega t .
$$

This is the form we shall need.

\section{ACKNOWLEDGMENTS}

This research was partially supported by the National Science Foundation under Grant INT-8504402 and by the U.S. Office of Naval Research under Contract N00014-86-K-0002.

Note added in proof. In [11], which presents a detailed discussion of the Langevin equation approach to dissipative problems, we discuss the kind of pitfalls that one must avoid in carrying out calculations involving a heat bath. 


\section{REFERENCES}

1. G. W. Ford, J. T. Lewis, And R. F. O'Connell, Phys. Rev. Lett. 55 (1985), 2273.

2. N. G. van Kampen, K. Dan. Vidensk. Selsk. Mat.-Fys. Medd. 26, No. 15 (1951).

3. A. MessiaH, "Quantum Mechanics," Vol. 2, Chap. 21, Sect. 21, North-Holland, Amsterdam, 1962.

4. G. W. Ford, J. T. Lewis, and R. F. O'Connell, Phys. Rev. A 36 (1987), 1466.

5. Bateman manuscript project. "Higher Transcendental Functions" (Erdélyi etal.), p. 18, McGraw-Hill, New York, 1953.

6. G. W. Ford, J. T. Lewis, and R. F. O'Connell, J. Phys. B 20 (1987), 899.

7. G. G. Barton, Phys. Rev. A 5 (1972), 468; J. Phys. B 20 (1987), 879.

8. H. B. Callen and T. A. Welton, Phys. Rev. 83 (1951), 34.

9. L. D. Landau and E. M. Lifshitz, "Statistical Physics," 3rd ed., Pergamon, Oxford. 1980.

10. K. M. CASE, Trans. Theory Statist. Phys. 2 (1972), 129.

11. G. W. Ford, J. T. Lewis, and R. F. O'Connell, Phys. Rev. A 37 (1988). 